\title{
Glueballs in Landau Gauge Yang-Mills theory from Dyson-Schwinger equations
}

\author{
Stefan Strauss* \\ Institut für theoretische Physik, \\ Justus-Liebig-Universität, \\ Heinrich-Buff-Ring 16, 35392 Gießen, \\ E-mail:stefan.strauss@theo.physik.uni-giessen.de
}

Christian S. Fischer

Institut für theoretische Physik,

Justus-Liebig-Universität,

Heinrich-Buff-Ring 16, 35392 Gießen,

E-mail: christian.fischer@theo.physik.uni-giessen.de

\section{Christian Kellermann}

Institut für Kernphysik,

Technische Universität Darmstadt,

Schlossgartenstraße 9, 64289 Darmstadt

E-mail: kellermannetheorie.ikp.physik.tu-darmstadt.de

\section{Lorenz von Smekal}

Institut für Kernphysik,

Technische Universität Darmstadt,

Schlossgartenstraße 9, 64289 Darmstadt

E-mail: lorenz.smekal@physik.tu-darmstadt.de

\begin{abstract}
We present results on the scalar glueball with $\mathrm{PC}=++$ in Landau gauge Yang-Mills theory within the Dyson-Schwinger/ Bethe-Salpeter equation approach. To this end, the gluon and ghost propagators are determined non-perturbatively in the whole complex momentum plane by numerical solutions of the respective Dyson-Schwinger equations. The resulting propagators are analytic apart from a cut structure on the real, timelike momentum axis and violate the OsterwalderSchrader positivity condition. The mass of the scalar glueball is obtained using a generalized ladder truncation to the Poincare-covariant Bethe-Salpeter equation.
\end{abstract}

Xth Quark Confinement and the Hadron Spectrum,

October 8-12, 2012

TUM Campus Garching, Munich, Germany

*Speaker. 


\section{Introduction}

The physical excitations in pure SU(N) Yang-Mills theory are color singlet glueballs [1]. There are various predictions for the glueball spectrum from different approaches $[2,3,4,5,6]$, making glueballs theoretically well-established. Experimentally, however, these states are difficult to identify since they mix with states containing pairs of quarks and antiquarks. The clarification of the unquenched glueball spectrum is therefore a prime theoretical task. Glueballs in pure Yang-Mills theory are, on the other hand, of considerable theoretical interest since they reflect the mass gap in the 'physical' spectrum of the theory. In this work we report on first results on this issue from the Dyson-Schwinger/ Bethe-Salpeter framework [7, 8].

In a linear covariant gauge, glueballs are bound states involving gluon and ghost degrees of freedom. In the simplest case this amounts to a superposition of a two-gluon bound state with a ghost-antighost component, which can be described by coupled set of Bethe-Salpeter equations. Key ingredients to such kind of approach are the non-perturbative propagators of the constituents at general complex momenta.

The non-perturbative complex gluon propagator is of course interesting in its own right. Color confinement is understood to be the absence of colored asymptotic states. Then a sufficient criterion for color confinement is a positivity violating spectral density. In a recent work [9] first numerical results for the propagators in the complex plane have been reported and negative contributions in the spectral functions have indeed been found confirming earlier evidence from Schwinger functions $[10,11]$.

This contribution is organized as follows: in the next section the Dyson-Schwinger equations (DSEs) for the ghost and gluon propagators and the ansaetze for the higher vertex functions are specified. In section 3 the well-known Euclidean solution is extended to complex momenta. Finally, a system of Bethe-Salpeter equations (BSEs) for glueballs as bound states of two gluons or two ghosts is sketched in the following section. We report a preliminary result for the lowest glueball mass, the $0^{++}$state.

\section{Yang-Mills Dyson-Schwinger equations}

In Landau gauge, the gluon and Faddeev-Popov ghost propagators are given by

$$
\begin{aligned}
D_{\mu v}(p) & =\left(\delta_{\mu v}-\frac{p_{\mu} p_{v}}{p^{2}}\right) \frac{Z\left(p^{2}\right)}{p^{2}}, \\
D_{G}(p) & =-\frac{G\left(p^{2}\right)}{p^{2}}
\end{aligned}
$$

with the gluon dressing function $Z\left(p^{2}\right)$ and the ghost dressing function $G\left(p^{2}\right)$. These are determined from their DSEs given diagrammatically in Fig. 1. The propagator DSEs are the lowest members of an infinite tower of coupled equations describing the one-particle irreducible Green's functions of a theory. In order to close the system at hand, one needs to specify the dressed ghostgluon, three-gluon and four-gluon vertices appearing in the loops on the right hand side of the equations. Currently there is great activity to include the vertex DSEs in the picture either from explicit calculations [12] or from systematic variations of ansaetze for the vertices explored on the 


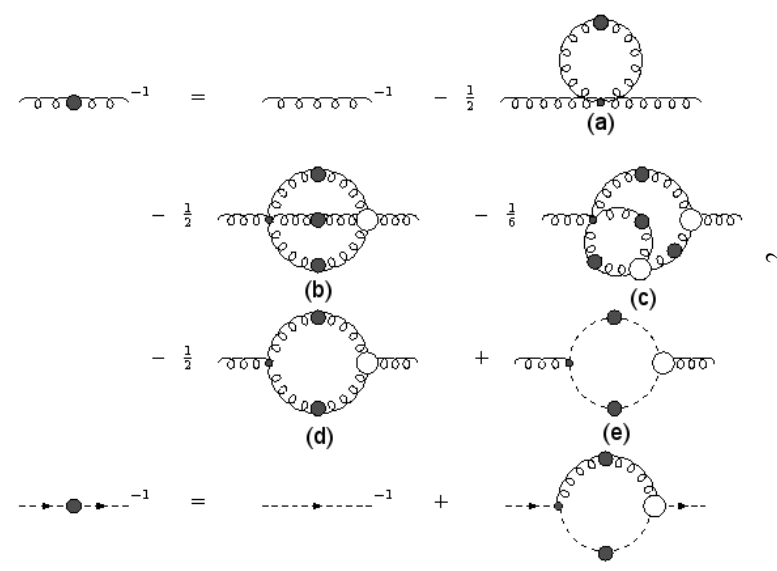

(a)

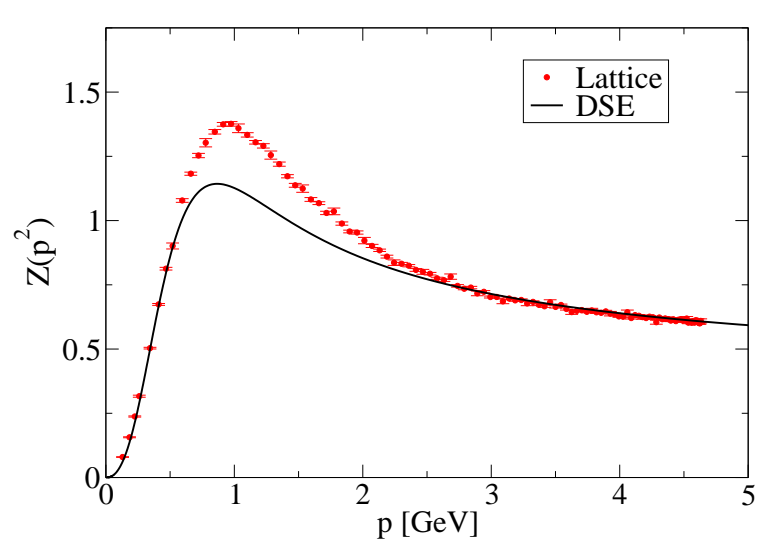

(b)

Figure 1: The Dyson-Schwinger equations for the ghost and gluon propagator in Landau gauge. Euclidean gluon dressing function compared with the lattice results of [13].

level of the propagator DSEs $[14,15]$. In this work we use the vertex ansaetze from Ref. [16] which explicitly read

$$
\begin{aligned}
\Gamma_{\mu}(p, q) & =\Gamma_{\mu}^{(0)}=i q^{\mu} \\
\Gamma_{\mu v \rho}(p, q) & =\frac{1}{Z_{1}} \frac{G^{-2 a}\left(q^{2}\right)}{Z^{a}\left(q^{2}\right)} \frac{G^{-2 a}\left((p-q)^{2}\right)}{Z^{a}\left((p-q)^{2}\right)} \Gamma_{\mu \nu \rho}^{(0)},
\end{aligned}
$$

where $\Gamma^{(0)}$ are the tree-level vertices and $a$ is related to the (perturbative) anomalous dimension of the ghost propagator. It is well known that this tree-level ansatz for the ghost-gluon vertex is a good qualitative approximation to the full ghost-gluon vertex $[17,18]$. The ansatz for the three-gluon vertex reproduces, by construction, resummed perturbation theory in the ultraviolet. Furthermore, contributions from the four-gluon vertex are neglected here (see however [19] for first results on the inclusion of the sunset diagram (b) in the left panel of Fig. 1). When compared with lattice calculations, the resulting solutions of the ghost-gluon system agree perfectly in the infrared and ultraviolet momentum region and deviate on the 20 percent level in the intermediate momentum regime, see the right panel of Fig. 1. There is also an active discussion on the deep infrared behavior of the gluon and ghost propagators. Two types of solutions, the scaling and decoupling one have been identified, which correspond to an infrared vanishing or finite gluon propagator $[11,20,21,22,23,24,25,26,27,28,29,30]$. In the following we focus on the decoupling solution.

\section{Analytic Structures of Gluons and Ghosts}

Traditionally, non-perturbative results of the gluon propagators have been limited to the spacelike momentum region, i.e. all computation have been done in Euclidean space-time for positive real squared momenta. Recovering the propagator also for time-like momenta requires either analytic continuation or solving the Yang-Mills DSE in the complex $p^{2}$ momentum plane. In [10] 




(a)

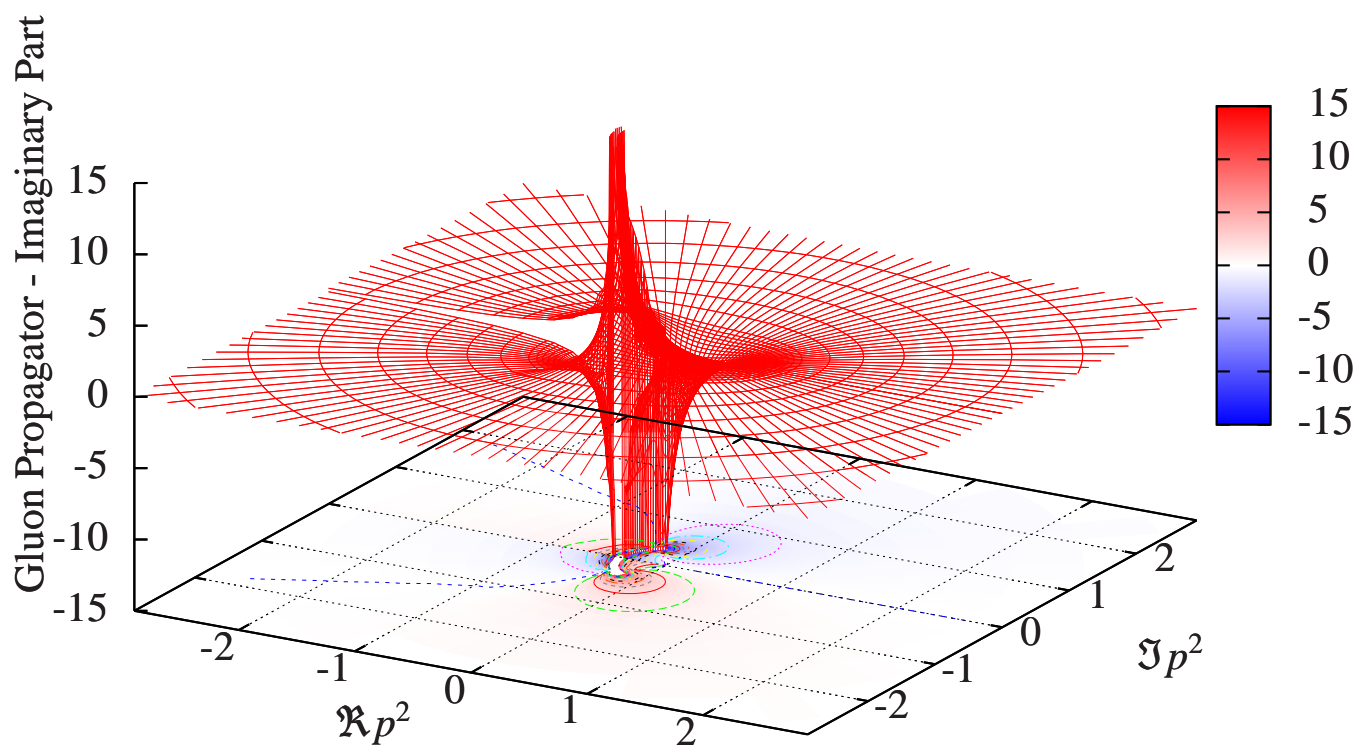

(b)

Figure 2: (Panel a) Results for the real part of the gluon propagator function $D\left(p^{2}\right)$. (Panel b) Results for the imaginary part of the gluon propagator function $D\left(p^{2}\right)$ [9]. The figures include colored contour maps and lines. The displayed range of the gluon propagator is restricted in order to resolve smaller structures.

the first method has been used and fit functions to the Euclidean propagators haven been continued analytically to complex momenta. In the case of the technically simpler fermion DSE, several methods for obtaining a numerical solution in the complex momentum plane have been devised in the past [31, 32]. In [9] the former method has been applied to the Yang-Mills DSEs for the first time.

The Figures 2 and 3 show the complex gluon propagator and the complex ghost dressing function, respectively [9]. Notice that both propagators have branch cuts, starting at the origin along 


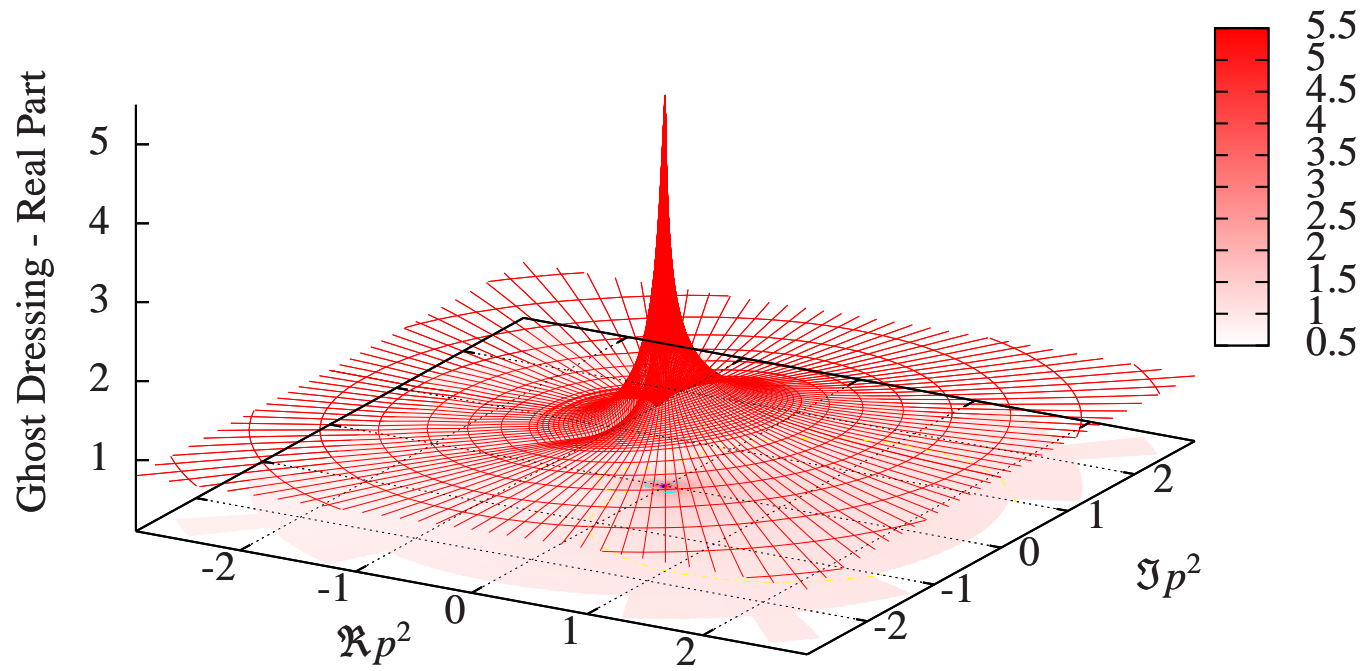

(a)

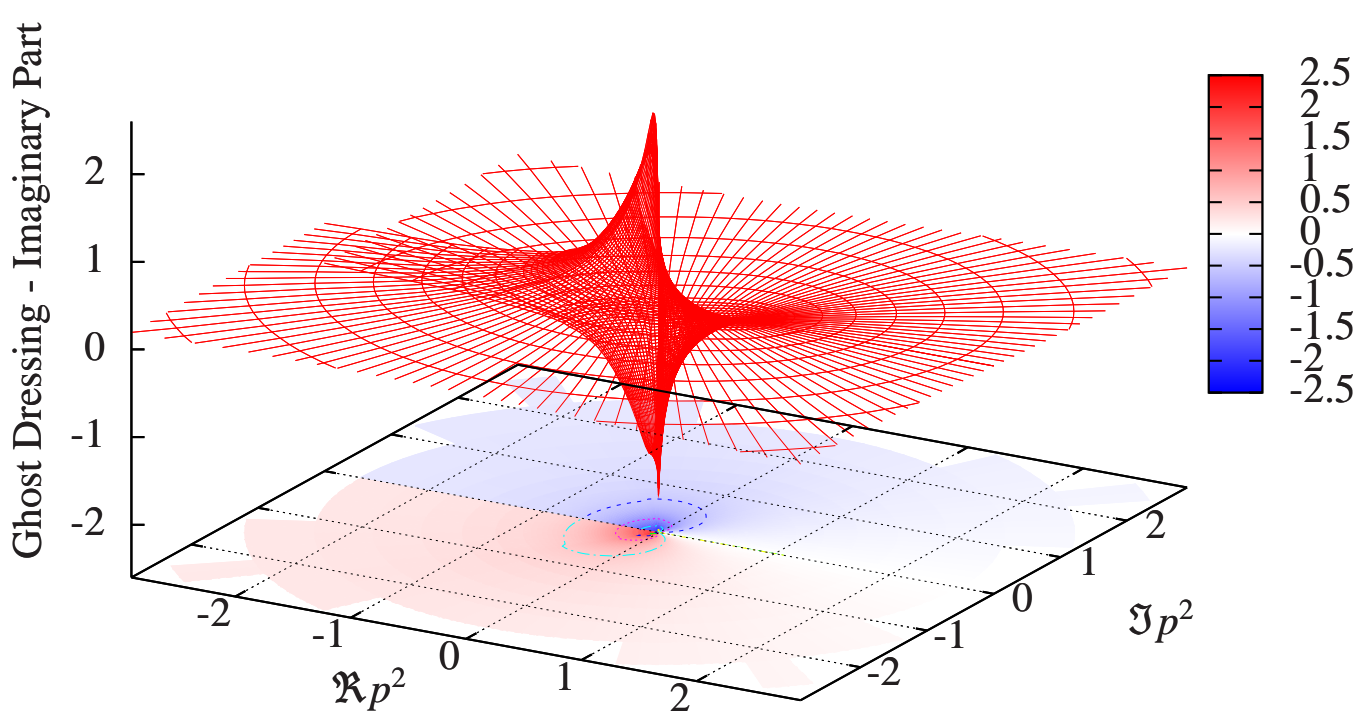

(b)

Figure 3: (Panel a) Results for the real part of the ghost dressing function $G\left(p^{2}\right)$. (Panel b) Results for the imaginary part of the ghost dressing function $G\left(p^{2}\right)$ [9]. The figures include colored contour maps and lines.

the negative real axis and extend to infinity. For the ghost propagator the branch cut is singular at zero. Moreover, the gluon propagator is highly peaked at certain regions of the complex plane but, within the present numerical accuracy, shows no additional singularities besides the branch cuts. This observation is in contrast to the suggested analytically forms for the gluon propagator in the literature $[33,34,35,36,37]$, which all assumed poles in the complex plane away from the real momentum axis. From our result we see no justification for these assumptions. Our result has a simple interpretation: the branch cuts correspond to the radiation of (massless) ghosts and gluons from the gluon via the ghost-gluon and three-gluon vertex couplings. Due to the positivity violations in the gluons and ghosts this radiation is one of unphysical particles into unphysical 
particles leaving no traces in the physical part of the state space of the theory. This part is occupied by the color-singlet glueball states discussed in the next section.

\section{Scalar Glueball}

Both DSEs and Bethe-Salpeter equations can be derived taking functional derivatives with respect to the propagators of a two-particle irreducible effective action (2PI EA) [38]. The general form of the 2PI EA is

$$
\Gamma\left[D, D_{G}\right]=\frac{1}{2} \operatorname{Tr} \ln \mathrm{D}_{0} \mathrm{D}^{-1}+\frac{1}{2} \operatorname{Tr} \mathrm{D}_{0}^{-1} \mathrm{D}-\operatorname{Tr} \ln \mathrm{D}_{\mathrm{G} 0} \mathrm{D}_{\mathrm{G}}^{-1}-\operatorname{Tr}_{\mathrm{G}_{0}}^{-1} \mathrm{D}_{\mathrm{G}}+\Gamma_{2}\left[\mathrm{D}, \mathrm{D}_{\mathrm{G}}\right],
$$

where $D$ and $D_{G}$ are the full gluon and ghost propagators and $D_{0}, D_{G 0}$ are their tree-level counterparts. Assuming the interaction $\Gamma_{2}$ diagrammatically as

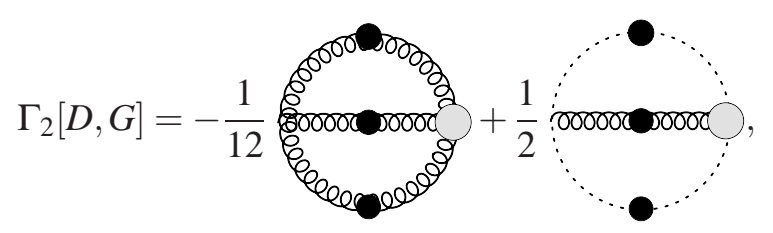

one finds the one loop DSEs for the ghosts and gluons, as discussed in Section 2. Applying two functional derivatives to (4.1) (while treating the vertices as explicit input) and demanding the two-particle onshell conditions provides the glueball BSEs

$$
\frac{\delta^{2} \Gamma}{\delta S \delta S} \gamma=0
$$

where $S$ stands for either the gluon or the ghost propagator and $\gamma$ is the Bethe-Salpeter amplitude. After introducing the modified Bethe-Salpeter amplitudes $\chi=S^{-1} \gamma S^{-1}$ one finds the following coupled system of BSEs for ghost and gluon bound states

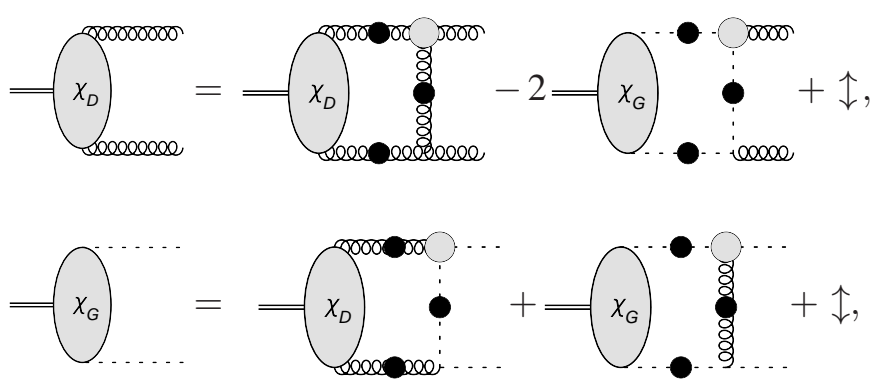

where the double arrow means symmetrization with respect to the dressed vertices. Note that the dressed vertices in the BSEs are the same as in the DSEs Eq. (2.2). Additionally, once the physical scale in the propagator equations is fixed there is no further adjustment of scales or coupling constants in the BSEs possible.

The scalar glueball gluon amplitude has the covariant representation

$$
\chi_{0^{++}}^{\mu v}(P, p)=A_{D}\left(p^{2}, p \cdot P\right) g^{\mu v}
$$

where $A_{D}$ denotes the dressing function. While the decomposition of glueball ghost amplitude reads

$$
\chi_{0^{++}}(P, p)=A_{G}\left(p^{2}, p \cdot P\right),
$$


where $P^{2}=M_{G}^{2}$ with $M_{G}$ being the glueball mass. Putting all ingredients together and solving the system of BSEs numerically we find a preliminary value for mass of the scalar glueball with $\mathrm{PC}=++$ of the order of

$$
m_{0^{++}}=1.8 \mathrm{GeV} .
$$

This result is in good agreement with findings from other approaches like lattice gauge theory [2, 3], Coulomb gauge Hamiltonians [4], Regge theory [5] and potential models [6].

\section{Conclusions}

In this contribution we have presented an approach to the glueball spectrum based on the BSE. These BSEs are a coupled system of equations describing glueballs as bound state of two gluons and two ghosts. As a first result the mass of the lowest scalar glueball was determined in agreement with several other theoretical approaches. A detailed account of our approach will be subject of a forthcoming publication [39].

Furthermore we summarized the results of [9] for the complex gluon and ghost propagators obtained by solving the corresponding DSEs for complex momenta. The complex dressing functions have no singularities besides branch cuts along the negative real axis. We do not observe pole singularities on the first Riemann sheet in the gluon propagator that are expected from the Gribov-Zwanziger approach or its modern counterparts. Both propagators have positivity violating spectral functions and allow no particle interpretation of gluons or ghosts as asymptotic states of the theory.

\section{Acknowledgments}

This work has been supported by the Helmholtz Young Investigator Grant VH-NG-332 and the Helmholtz International Center for FAIR within the LOEWE program of the State of Hesse.

\section{References}

[1] H. Fritzsch and P. Minkowski, Nuovo Cim. A 30 (1975) 393.

[2] G. S. Bali et al. [UKQCD Collaboration], Phys. Lett. B 309 (1993) 378 [hep-lat/9304012].

[3] C. J. Morningstar and M. J. Peardon, Phys. Rev. D 60 (1999) 034509 [hep-lat/9901004].

[4] A. P. Szczepaniak and E. S. Swanson, Phys. Lett. B 577 (2003) 61 [hep-ph/0308268].

[5] F. J. Llanes-Estrada, P. Bicudo and S. R. Cotanch, Phys. Rev. Lett. 96 (2006) 081601 [hep-ph/0507205].

[6] F. Brau and C. Semay, Phys. Rev. D 70 (2004) 014017 [hep-ph/0412173]; V. Mathieu, C. Semay and B. Silvestre-Brac, Phys. Rev. D 77 (2008) 094009 [arXiv:0803.0815 [hep-ph]].

[7] R. Alkofer, L. von Smekal, Phys. Rept. 353 (2001) 281. [hep-ph/0007355].

[8] C. S. Fischer, J. Phys. G G 32 (2006) R253 [hep-ph/0605173].

[9] S. Strauss, C. S. Fischer and C. Kellermann, Phys. Rev. Lett. 109 (2012) 252001 [arXiv:1208.6239 [hep-ph]]. 
[10] R. Alkofer, W. Detmold, C. S. Fischer and P. Maris, Phys. Rev. D 70 (2004) 014014 [hep-ph/0309077]; Nucl. Phys. Proc. Suppl. 141 (2005) 122 [hep-ph/0309078].

[11] C. S. Fischer, A. Maas, J. M. Pawlowski, Annals Phys. 324 (2009) 2408-2437.

[12] M. Q. Huber and L. von Smekal, arXiv:1211.6092 [hep-th].

[13] A. Sternbeck, E. M. Ilgenfritz, M. Muller-Preussker, A. Schiller and I. L. Bogolubsky, PoS LAT2006, 076 (2006) [arXiv:hep-lat/0610053].

[14] M. R. Pennington and D. J. Wilson, Phys. Rev. D 84 (2011) 119901 [arXiv:1109.2117 [hep-ph]].

[15] P. .Boucaud, D. Dudal, J. P. Leroy, O. Pene and J. Rodriguez-Quintero, JHEP 1112 (2011) 018 [arXiv:1109.3803 [hep-ph]].

[16] C. S. Fischer, R. Alkofer, Phys. Lett. B 536 (2002) 177.

[17] W. Schleifenbaum, A. Maas, J. Wambach, R. Alkofer, Phys. Rev. D72 (2005) 014017. [hep-ph/0411052].

[18] A. Cucchieri, A. Maas, T. Mendes, Phys. Rev. D77 (2008) 094510. [arXiv:0803.1798 [hep-lat]]..

[19] V. Mader and R. Alkofer, contribution in this volume

[20] C. Lerche, L. von Smekal, Phys. Rev. D 65 (2002) 125006.

[21] J. M. Pawlowski, D. F. Litim, S. Nedelko and L. von Smekal, Phys. Rev. Lett. 93, 152002 (2004). C. S. Fischer and J. M. Pawlowski, Phys. Rev. D 75, 025012 (2007); Phys. Rev. D80 (2009) 025023.

[22] R. Alkofer, C. S. Fischer and F. J. Llanes-Estrada, Phys. Lett. B 611, 279 (2005);

[23] A. C. Aguilar, D. Binosi and J. Papavassiliou, Phys. Rev. D 78 (2008) 025010.

[24] Ph. Boucaud, et al. JHEP 0806, 012 (2008); JHEP 0806, 099 (2008).

[25] A. Maas, Phys. Lett. B689 (2010) 107.

[26] L. von Smekal, arXiv:0812.0654 [hep-th].

[27] A. Sternbeck, L. von Smekal, Eur. Phys. J. C68 (2010) 487; A. Maas et. al, Eur. Phys. J. C 68 (2010) 183.

[28] A. Cucchieri and T. Mendes, Phys. Rev. D 78 (2008) 094503; Phys. Rev. Lett. 100, 241601 (2008). I. L. Bogolubsky et al., Phys. Lett. B676 (2009) 69.

[29] A. Cucchieri and T. Mendes, Phys. Rev. D 81 (2010) 016005 [arXiv:0904.4033 [hep-lat]].

[30] A. Sternbeck and M. Muller-Preussker, arXiv:1211.3057 [hep-lat].

[31] P. Maris, Phys. Rev. D 52 (1995) 6087 [hep-ph/9508323].

[32] C. S. Fischer, D. Nickel and R. Williams, Eur. Phys. J. C 60 (2009) 47 [arXiv:0807.3486 [hep-ph]].

[33] V. N. Gribov, Nucl. Phys. B 139 (1978) 1.

[34] D. Zwanziger, Nucl. Phys. B 323 (1989) 513.

[35] M. Stingl, Phys. Rev. D 34 (1986) 3863 [Erratum-ibid. D 36 (1987) 651].

[36] A. Cucchieri, D. Dudal, T. Mendes and N. Vandersickel, Phys. Rev. D 85 (2012) 094513 [arXiv:1111.2327 [hep-lat]].

[37] D. Dudal, J. A. Gracey, S. P. Sorella, N. Vandersickel and H. Verschelde, Phys. Rev. D 78, 065047 (2008).

[38] D. W. McKay and H. J. Munczek, Phys. Rev. D 40 (1989) 4151.

[39] C. S. Fischer, C. Kellermann, L. von Smekal and S. Strauss, in preparation. 